\title{
Carcinoma Microinvasor de Cuello Uterino
}

\author{
Drs.: laime Botero Uribe*, Jaime Uribe Duque** y Juan Luis Londoño***
}

\section{INTRODUCCION}

El presente estudio constituye una revisión retrospectiva de los casos de carcinoma microinvasor de cuello uterino que se han presentado en el servicio de Oncología del Hospital Universitario San Vicente de Paúl de Medellín desde el 10. de enero de 1970 hasta el 31 de diciembre de 1984 y busca encontrar orientaciones que nos permitan adoptar para el futuro una modalidad de tratamiento que a la vez que ofrezca la máxima seguridad a las pacientes, utilice en forma óptima los recursos médicos y permita un seguimiento adecuado para determinar la eficacia a largo plazo de las medidas terapéuticas.

El término carcinoma microinvasor o microcarcinoma del cuello uterino fue introducido por Mestwerdt en 1947 y

* Profesor Emérito, Departamento de Obstetricia y Ginecología, Facultad de Medicina, Universidad de Antioquia.

** Profesor Titular, Departamento de Obstetricia y Ginecología, Facultad de Medicina, Universidad de Antioquia.

*** Profesor Titular, Facultad Nacional de Salud Pública, Universidad de Antioquia. desde entonces los intentos para definirlo adecuadamente han resultado confusos y arbitrarios. La definición, sin embargo, tiene importancia por las implicaciones que de ella se derivan para la elección del tratamiento y su ulterior evaluación, La última adoptada por la FIGO subdivide la entidad en estadio $\mid \mathrm{A} 1$, que es invasión incipiente o inicial del estroma, y estadio IA2 que es invasión por debajo de la membrana basal con máximo de $5 \mathrm{~mm}$. y una extensión superficial de $7 \mathrm{~mm}$. Parece, sin embargo, que esta definición es de difícil aplicación y no refleja adecuadamente los principales criterios pronósticos que se han identificado hasta el presente.

Los factores que más se han prestado a confusión hasta el momento se pueden resumir en: profundidad de penetración estromal, compromiso de microlinfáticos y/o espacios similares a capilares por el tumor, volumen del mismo, y otros criterios histológicos propios de los tumores invasores tales como confluencia de la masa tumoral y diferenciación del epitelio (1).

Con relación al primero de ellos, la profundidad de penetración en el estroma, los estudios se han dirigido principalmente a determinar a partir de qué dis- 
tancia empieza a ocurrir el hallazgo de ganglios positivos en la pelvis. Si se tiene en cuenta que la mortalidad quirúrgica consecutiva a la histerectomía radical con disección linfática es de 0.6 al $1.2 \%$, la realización de tal procedimiento sólo se justifica si las posibilidades de hallar ganglios positivos son mayores del 1 al $2 \%$. Una revisión crítica de la literatura realizada por Benson y Norris muestra las dificultades que existen para juzgar los resultados que presentan varios investigadores, principalmente por el número reducido de casos que han podido estudiar $y$ en algunas ocasiones debido a que no se hace mención del número de ganglios hallados durante la intervención quirúrgica (2).

En las tablas Nos. 1 y 2 tomadas de van Nagell et al (3) se observa la frecuencia de metástasis a los ganglios linfáticos según si la penetración estromal es hasta de $3 \mathrm{~mm}$. o entre 3 y $5 \mathrm{~mm}$. De 145 pacientes en quienes la invasión al estroma era de $3 \mathrm{~mm}$. o menos y que fueron seguidos después del tratamiento en el Centro Médico de la Universidad de Kentucky, por un promedio de 7.2 años, solamente en dos casos se presentó recurrencia del tumor, de la variedad in situ, en el vértice de la vagina y ambos

CANCER MICROINVASOR DE CUELLO UTERINO

1970 - 1984

TABLA I-FRECUENCIA DE METASTASIS A gANGLIOS LINFATICOS EN PACIENTES CON INVASION AL ESTROMA MENOR O IGUAL A 3 MM

\begin{tabular}{|c|c|c|}
\hline ESTUDIO & $\begin{array}{l}\text { NE DE } \\
\text { PACIENTES }\end{array}$ & $\begin{array}{l}\text { NE DE PACIENTES } \\
\text { CON GGLIOS POS }\end{array}$ \\
\hline SMITH FOUSHEE ET AL (1969) & 16 & 0 \\
\hline ROCHE NORRIS (1975) & 9 & 0 \\
\hline LEMAN ET AL $(1976)$ & 32 & 0 \\
\hline SESKI ET AL (1977) & 37 & 0 \\
\hline TAKI ET AL (1979) & 55 & 0 \\
\hline YAJIMA O NODA (1979) & 90 & 0 \\
\hline HASUMI ET AL $(1980)$ & 106 & 1 \\
\hline VAN NAGELL ET AL (1982) & 52 & 0 \\
\hline TOTAL & 397 & 1 \\
\hline
\end{tabular}

Fuente $=$ Rot $* 3$
CANCER MICROINVASOR DE CUELLO UTERINO

$1970-1984$

TABLA 2-FRECUENCIA DE METASTASIS A GANGLIOS LINFATICOS

EN PACIENTES CON INVASION ESTROMAL DE 3.1 A 5 MM

\begin{tabular}{|c|c|c|}
\hline ESTUDIO & $\begin{array}{l}\text { N2 OE } \\
\text { PACIENTES }\end{array}$ & $\begin{array}{l}\text { NE DE PACIENTES } \\
\text { CON GBLIOS POS }\end{array}$ \\
\hline SMITH FOUSHEE ET AL (1969) & 13 & 1 \\
\hline $\begin{array}{l}\text { ROCHE \& NORRIS }(1975) \\
\text { LEMAN ET AL }(1976)\end{array}$ & $\begin{array}{r}21 \\
3\end{array}$ & $\begin{array}{l}0 \\
0\end{array}$ \\
\hline HASUMI ET AL $(1980)$ & 29 & 4 \\
\hline VAN NAGELL ET AL (1982) & 32 & 3 \\
\hline TOTAL & 98 & 8 \\
\hline
\end{tabular}

Fuente = Ref $* 3$

fueron curados. En 32 casos en los cuales se presentó invasión a una profundidad entre 3.1 y $5 \mathrm{~mm}$. se encontraron metástasis en 3 casos y dos de ellos murieron por carcinoma invasor. En su estudio, parece que la invasión vascular o linfátic: tiene mayor significación en las paciente que tienen infiltración mayor de $3 \mathrm{~mm}$. Proponen como definición que puede ser útil aquella que muestra invasión del estroma de $3 \mathrm{~mm}$. o menos, sin invasión a espacios vasculares o linfáticos, y recomiendan estudiar un número mayor de pacientes para determinar si el compromiso vascular y linfático y la penetración al mismo tiempo menor de $3 \mathrm{~mm}$. se pueden considerar aún como carcinoma microinvasor.

En algunos estudios sobre carcinoma de cuello realizados en distintas ciudades de Colombia, se encuentra que el hallazgo de lesión microinvasora es poco frecuente, como se ve en la tabla No. 3.

El presente estudio pues, hace parte de uno más amplio que pretende buscar orientaciones similares para el carcinoma de cuello uterino estadio lb, el carcinoma de ovario, de endometrio y de vulva y será presentado para discusión en el XVI Congreso Colombiano de Obstetricia y Ginecología en diciembre del presente año. 
CANCER MICROINVASOR DE CUELLO UTERINO

$1970-1984$

TABLA 3-HALLAZGO DE CANCER MICROINVASOR EN ESTUDIOS

SOBRE CANCER DE CUELLO REALIZADOS EN COLOMBIA

\begin{tabular}{|lclc|}
\hline \multicolumn{1}{|c}{ SITIO } & AÑO & $\begin{array}{c}\text { NUMERO Y TIPO DE } \\
\text { CASOS }\end{array}$ & $\begin{array}{c}\text { CA } \\
\text { MICROINYASOR }\end{array}$ \\
\hline IBAGUE (4) & $1974-76$ & 80, IN SITU E INV & 2 \\
CALI (5) & $1970-76$ & 119 , IN SITU & 5 \\
BOGOTA (6) & $1966-75$ & 198 CONIZACIONES & 9 \\
BOGOTA, MEDELLIN, & & \\
CARTAGENA (7) $1972-81$ & 152, EMBARAZADAS & 6 \\
\hline
\end{tabular}

Hemos estudiado en forma retrospectiva las historias clínicas de 103 pacientes que figuran en el registro de cáncer ginecológico con el diagnóstico de carcinoma microinvasor del cuello uterino, para analizar algunos hechos que creemos que pueden tener utilidad para entender mejor el comportamiento de esta enfermedad entre nosotros, para ver la efectividad del tratamiento y la asiduidad conque las pacientes acuden a la consulta de seguimiento.

Lo que más nos ha llamado la atención en este estudio es que, a pesar de las dificultades que se tienen para el seguimiento de estas pacientes, dadas sus condiciones socio-culturales y los sitios alejados en donde viven, se ha conseguido uno muy satisfactorio en más del $80 \%$ de los casos, lo cual permitirá una mejor evaluación de los procedimientos terapéuticos que se están empleando $y$ que se definan para el futuro. Hemos encontrado por otra parte, que los estudios de la primera biopsia no definen la profundidad de penetración de la lesión en el estroma, y los del espécimen quirúrgico tampoco lo hacen con la frecuencia deseable. No se habla, sino en muy pocos casos, del compromiso de los espacios vasculares. El estudio definitivo del espécimen quirúrgico demostró que había invasión franca en un $18 \%$ de los casos en que inicialmente se pensó que se trataba de un carcinoma microinvasor, por lo cual fue necesario aplicar tratamientos adicionales, principalmente radioterapia $y$ en algunos cirugía radical. Cuando se tomó la biopsia a la gran mayoría de estas pacientes no se tenía aún el recurso de la colposcopia. Qué tanta influencia tuvo en que ocurrieran estos hechos la demora entre la toma de la primera biopsia $y$ el tratamiento definitivo, que en una gran proporción de casos se extendió a varios meses, es difícil de determinar en este estudio.

\section{MATERIAL Y METODOS}

La población seleccionada está constituida por las pacientes atendidas en el servicio de Oncología del Hospital Universitario San Vicente de Paúl de MedeIlín entre el 1o. de enero de 1970 y el 31 de diciembre de 1984, en quienes se hizo el diagnóstico de carcinoma microinvasor de cuello uterino mediante estudio de biopsia. Los casos se tomaron de un archivo de datos del tipo Applework llevado mediante el uso de un rnicro. computador marca Apple lle y la información que aquí se presenta fue extraída en parte del archivo mencionado $y$ en parte de las historias clínicas de las pacientes, que reposan en la sección correspondiente del Hospital.

\section{RESULTADOS}

Están consignados en los gráficos 1 a 4 y en las tablas 4 a 7 . Llama la atención la alta proporción de mujeres jóvenes, ya que más de un 12\% están por debajo de los 30 años y la mayor parte tienen menos de 50 .

Es muy parca la descripción histológica tanto de las biopsias como de los co- 


\section{CANCER MICROINVASOR DE CUELLO UTERINO}

GRAFICA I-DISTRIBUCION POR GRUPOS DE EDAD DE LAS PACIEN-

TES CON DIAGNOSTICO DE CANCER MICROINVASOR

$1970-1984$

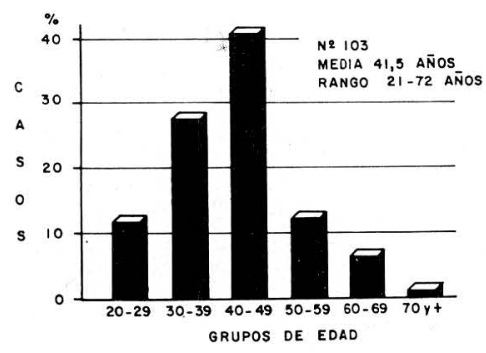

nos y de los especímenes quirúrgicos totales, ya que raramente se menciona la profundidad de penetración de la lesión y el compromiso de espacios vasculares sanguíneos y linfáticos.

CANCER MICROINVASOR DE CUELLO UTERINO

$1970-1984$

GRAFICA 2 - TIPOS DE TRATAMIENTO QUIRURGICO

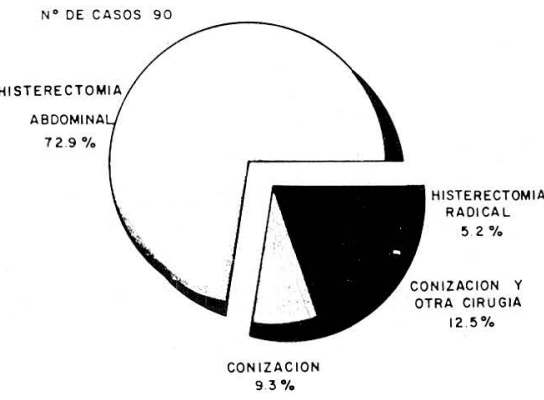

La histerectomía abdominal fue el tratamiento de elección y la conización sola ocupó un lugar bajo, con menos del $10 \%$ de los casos.

Obsérvese en el gráfico 3 la proporción de casos en los cuales se encontró carcinoma invasor en el espécimen definitivo, que hizo que se tuviera que complementar el tratamiento agregando una cirugía radical.
CANCER MICROINVASOR DE CUELLO UTERINO $1970-1984$

GRAFICA 3-HALLAZGOS DEL ESPECIMEN QUIRURGICO, CON. DIAGNOSTICO EN LA BIOPSIA DE CO

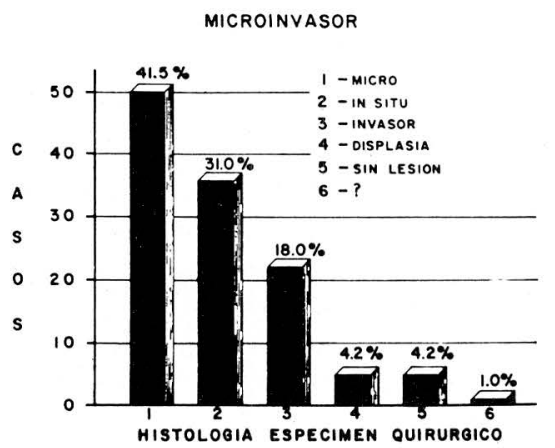

El tiempo transcurrido entre la toma de la biopsia inicial y el tratamiento definitivo, fue muy variable $y$ en muchos casos se extendió a varios meses, lo cual constituye un riesgo grande para una variedad de tumor en la que una penetración de unos pocos $\mathrm{mm}$ puede hacer cambiar el pronóstico.

CANCER MICROINVASOR DE CUELLO UTERINO GRAFICA 4 - TIEMPO QUE TRANSCURRIO EN DIAS ENTRE LA TOMA DE

LA PRIMERA BIOPSIA Y EL TRATAMIENTO

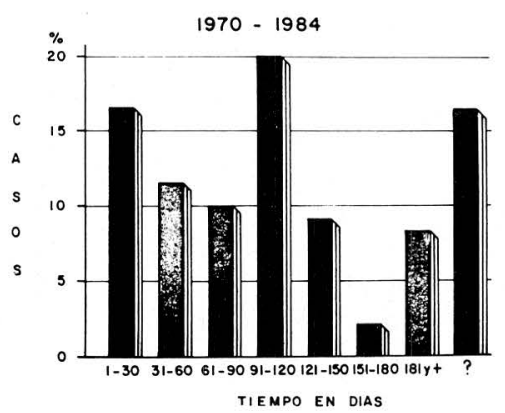

Los demás resultados son fácilmente observables y se destaca principalmente la demora entre la toma de la biopsia inicial y el tratamiento, que en muchos casos se extendió a varios meses. Por lo 
CANCER MICROINVASOR DE CUELLO UTERINO

$1970-1984$

TABLA 4 - PROFUNDIDAD DE PENETRACION EN EL ESTROMA Y COMPROMISO LINFATICO EN LA BIOPSIA

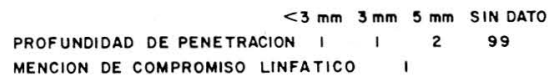
CANCER MICROINVASOR DE CUELLO UTERINO $1970-1984$

TABLA 5- INDICACIONES PARA LA CONIZACION

\begin{tabular}{|lcc|}
\hline INDICACION & CASOS & PORCENTAJE \\
\hline DIAGNOSTICO & 9 & 0.73 \\
TRATAMIENTO & 14 & 13.59 \\
SIN CONIZACION & 80 & 85.68 \\
\hline TOTAL & $\frac{80}{103}$ & $\frac{10000}{}$ \\
\hline
\end{tabular}

demás, es muy satisfactorio el hallazgo de una mortalidad tan baja, pues es de $0 \%$ a los 5 años, y de un seguimiento que en nuestro medio se considera muy satisfactorio.

CANCER MICROINVASOR DE CUELLO UTERINO

$1970-1984$

TABLA 6 - SEGUIMIENTO RECURRENCIAS Y SOBREVIDA

\begin{tabular}{|lcc|}
\hline TOTAL IO3 CASOS & CASOS & PORCENTAJE \\
\hline MURIERON DURANTE EL INTERVALO & 2 & 1.94 \\
RECURRENCIAS & 2 & 1.94 \\
PERDIDAS DE SEGUIMIENTO & 18 & 17.47 \\
\hline
\end{tabular}

CANCER MICROINVASOR DE CUELLO UTERINO SUPERVIVENCIA A 5 AÑOS
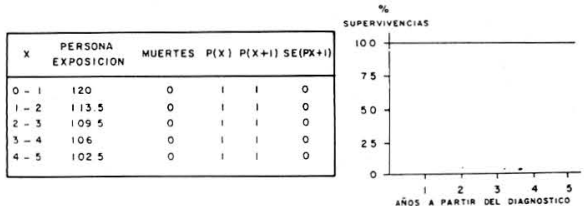

\section{DISCUSION}

Se destaca como hecho importante en este análisis lo escaso que fue el estudio detallado del espécimen quirúrgico, que junto con lo poco frecuente que fue la obtención de conos para la aclaración de los diagnósticos y con la ausencia de estudio, colposcópico en la mayoría de las pacientes, creemos que influyó para que se hubiera encontrado una proporción tan alta de casos de carcinoma invasor al estudiar el espécimen definitivo.

Es también muy digno de destacarse el tiempo que transcurrio entre la toma de la biopsia y el tratamiento definitivo. Si comparamos esa demora en los casos de carcinoma microinvasor con la que ocurrió en el invasor, encontramos que en el $54.2 \%$ de los micros el tratamiento se demoró más de 60 días, mientras que para los invasores francos esa demora ocurrió en el $68.7 \%$ de los casos. Si tomamos como de un mes el intervalo para el tratamiento definitivo una vez tomada la biopsia, que aún es susceptible de reducirse, vemos como en el $82.5 \%$ de los micros fue mayor de ese tiempo, mientras que en el caso de los invasores lo fue en el $93.7 \%$ de los casos.

Se hace pues énfasis en la necesidad de una mejor descripción histológica que ayude a una definición más precisa del tratamiento que mejor conviene a la paciente. Esto es importante, como se vio en la introducción, para efectuar un tratamiento más acorde con la extensión de la lesión con el fin de evitar riesgos innecesarios. Esto, unido a una mayor frecuencia en la realización de conizaciones diagnósticas, creemos que puede hacer más precisos los tratamientos y evitar los riesgos inherentes a los más radicales, el uso de la radioterapia posterior a la ci- 
rugía y naturalmente la reducción en los costos de atención a las pacientes, que es una consideración importante desde que no repercuta en disminución de los porcentajes de curación.

Creemos que lo anterior permitirá explorar nuevas modalidades de tratamiento, que siendo igualmente eficaces resulten en menores riesgos e incapacidad para las pacientes y en menores costos para el hospital.

Es de la mayor importancia utilizar adecuadamente la infraestructura de seguimiento que está en funcionamiento en el servicio de Oncología Ginecológica para aprovechar al máximo el tiempo y disminuir el intervalo entre la primera biopsia y el tratamiento, que en todo caso no debe ser mayor que el estrictamente indispensable para el estudio adecuado y completo de los cortes histológicos y para la ejecución de los exámenes prequirúrgicos que estén indicados, so pena de ver que algunos casos avancen a estadios que van a hacer cada vez más difícil la curación.

\section{BIBLIOGRAFIA}

1. MATTINGLY, R. Moderator's summary. Microinvasive Carcinoma. III World Congress for Cervical Pathology and Colposcopy. Orlando, Florida. Obstet. Gynecol. Surv. 34: 841-843, 1979.

2. BENSON, W.L. \& NORRIS, H.J. A critical review of the frequency of lymph node metastasis and death from microinvasive carcinoma of the cervix. Obstet. 49 (5): 632-638. May, 1977.

3. VAN NAGELL, J.R., GRENWELL, N., POWELL, D.F., DONALDSON, E.S., HANSON, M.B. \& GAY, E.C. Microinvasive carcinoma of the cervix. Am. J. Obstet. Gynecol. 145 (8): 981-991. April 1983.

4. RENGIFO, J. Carcinoma de cuello uterino. Revisión y manejo. Rev. Colombiana Obstet. Ginecol. 27 (2): 73-83, MarzoAbril, 1977. 
5. AMOROCHO, N.H. \& HENAO, F.: Carcinoma in situ. Diagnóstico $\mathrm{y}$ manejo. Biopsia frente a cono. Rev. Colombiana Obstet. Ginecol. 27 (6): 233-240, Nov. Dic., 1977.

6. ROMERO, J. DEL C.: La conización del cervix. Rev. Colombia Obstet. Ginecol. 30 (4): 151-159, Jul. Agos., 1979.

7. CARDONA, F., ACEVEDO, G., QUIJANO, H., PARDO, F., RODRIGUEZ M.,
URIBE, J., SOTO, A. \& BARRIOS, J.: Diagnóstico $y$ tratamiento de las neoplasias interepiteliales $y$ de los carcinomas cervicales invasores en el embarazo. Rev. Colombiana Obstet. Ginecol. 34 (4), 211 220, Jul. Agos. 1983.

8. LARSSON, G.: Conización for preinva sive and early invasive carcinoma of the uterine cervix. Acta Obstet. Gynecol. Scand. 43, Suppl. 114, 1983. 